\title{
Die nächste \\ Generation präsentiert
}

In insgesamt 15 Jahren auf dem Markt hat sich das Verankerungssystem LOCATOR des amerikanischen Traditionsunternehmens ZEST Anchors zum führenden Standard für implantatgestützte Prothesen entwickelt. Ab sofort ist mit dem ZEST LOCATOR R-Tx die nächste Generation des bewährten Verankerungssystems erhältlich - ein einfacheres, stärkeres und besseres System, das auf die gleiche Versorgungsform wie sein Vorgänger setzt.

Die neuartige, exklusive DuraTec ${ }^{\mathrm{TM}}$ Beschichtung aus Titancarbonitrid optimiert nicht nur die Ästhetik, sondern steigert auch Härte und Abrasionsresistenz. Zudem wurde der Schraubmechanismus vereinfacht: Während der bewährte LOCATOR spezielles Werkzeug erfordert, kommt beim LOCATOR R-Tx ein industriestandardisiertes Tool mit .050“/1,25mm* Sechskant-Schraubmechanismus zum Einsatz. Weitere Vorteile des neuen Systems: Duale Retentionselemente beim Abutment und bei den Nylon-Retentionseinsätzen harmonisieren mit dem optimierten Matrizengehäuse und sorgen für eine um 50 \% höhere Schwenkkapazität (mit bis $\mathrm{zu} 60^{\circ}$ zwischen Implantaten), wodurch der Patient die Prothese leichter ausrichten und punktgenau einsetzen kann. Darüber hinaus weist das neu designte Matrizengehäuse in anodisiertem Pink horizontale Rillen und Flächen auf, die für mehr Widerstand gegenüber vertikalen und horizontalen Bewegungen sorgen. Anwenderfreundlich ist auch die praktische, doppelseitige All-InOne-Verpackung, die das Abutment und die für die Verarbeitung notwendigen Komponenten separat beinhaltet und so alle erforderlichen Komponenten unter einer Artikelnummer bereitstellt. 